\title{
SIMULAÇÃO EM VIDEOLAPAROSCOPIA: UMA INOVAÇÃ̃O PARA ACADÊMICOS DE MEDICINA
}

Simlation in videolaparoscopy: na ainnovation for medicine academics

Simulación em videolaparoscopía: una innovación para la academia em la medicina

\section{Jarissa Mayara Ducarmo da Silva Soares ${ }^{* 1}$, Janderson da Silva Soares ${ }^{2}$, ${ }^{3}$ Marcelo Mendes Ribeiro ${ }^{3}$}

${ }^{1}$ Curso de Medicina, Universidade de Gurupi, Gurupi, Brasil.

${ }^{2}$ Laboratório de Ciências de la salud, Curso de Medicina, Universidade Central Del Paraguai, Pedro Juan Caballero, Paraguai.

${ }^{3}$ Cirurgião Geral, Universidade Federal do Vale do São Francisco, Petrolina, Brasil

*Correspondência: Universidade de Gurupi - Endereço: Avenida Guanabara $N^{o} 1308$, esquina com a Rua Ministro Alfredo Nasser,CEP: 77403080 Email: jarissasoares10@gmail.com

Artigo recebido em 20/11/2020 aprovado em 22/06/2021 publicado em 05/11/2021

\section{RESUMO}

Objetivo: desenvolver modelo de treinamento em vídeo-cirurgia, como fonte geradora de imagem para um aperfeiçoamento do acadêmico de medicina. Metodologia: Utilizou-se de uma pesquisa experimental aberta, com 30 participantes ativos, comparando suas habilidades de grupo em tempo e destrezas com materiais. Resultados: Nestes exercícios de simulação encontramos, melhora das habilidades nos exercícios e melhora do tempo quanto a destreza dos participantes. Conclusão: Um bom cirurgião em vídeolaparoscopia requer habilidade e uma visão bidimensional devido a limitação dos movimentos no emprego do método. Os alunos alcançaram habilidades mais cedo sem necessidade de muitas repetições nos exercícios.

Palavras-chave:Videolaparoscopia, Modelos de laparoscopia, Inovação cientifica.

\section{ABSTRACT}

Objective: todevelop a training model in videosurgery, as animagegeneratingsource for theimprovementof medical students. Methodology: An open experimental researchwasused, with30 activeparticipants, comparingtheirgroupskills in time andskillswithmaterials. Results: In thesesimulationexercises, wefoundanimprovement in theskills in theexercisesandanimprovement in time regardingtheparticipants' dexterity. Conclusion: A goodsurgeon in video-laparoscopyrequiresskilland a two-dimensional viewduetolimitedmovementwhenusingthemethod. Studentsachievedskillsearlierwithoutrequiringmanyrepetitions in theexercises.

Keywords: Videolaparoscopy, Laparoscopymodels, Scientificinnovation.

\section{RESUMEN}

Objetivo: desarrollar un modelo de capacitación en video cirugía, como fuente generadora de imágenes para la mejora de los estudiantes de medicina. Metodología: Se utilizó una investigación experimental abierta, con 30 participantes activos, comparando sus habilidades grupales en tiempo y habilidades con materiales. Resultados: En estos ejercicios de simulación, encontramos una mejora en las habilidades en los ejercicios y una mejora en el tiempo con respecto a la destreza de los participantes. Conclusión: Un buen cirujano en video-laparoscopia requiere habilidad y una vista bidimensional debido al movimiento limitado cuando se utiliza el método. Los estudiantes lograron habilidades antes sin requerir muchas repeticiones en los ejercicios.

Descriptores: Videolaparoscopia, Modelos de laparoscopia, Innovación científica. 


\section{INTRODUÇÃO}

A simulação surge como um instrumento complementar ao treinamento tradicional para a aquisição de habilidades cirúrgicas, possibilitando encurtar as curvas de aprendizado em um ambiente seguro e controlado (Reznick et al, 2006; Aggarwal; Darzi, 2011). Alguns estudos demonsta que aproximadamente 2 horas de um programa de simulação virtual (derivado da realidade virtual, que dá ao usuário a sensação de estar presente nele) é igual a uma hora de voo real (Morrison; Hammon, 2000). Essa experiência de longa duração pode ser proposta para o ensino de cirurgia, particularmente a minimamente invasiva.

$\mathrm{O}$ treinamento em simulação oferece a oportunidade de aprender em um ambiente estruturado e eficiente, sem comprometer a segurança do paciente. Permite que todos os alunos tenham acesso igual a cenários clínicos fictícios que incentivem a prática deliberada e repetitiva do procedimento (Ericsson, 2008), juntamente com uma avaliação padronizada e monitorada, com objetivos claros previamente definidos. Por fim, a simulação permite feedback efetivo do educador

A laparoscopia revolucionou a cirurgia nas últimas décadas. As cirurgias passaram a ser realizadas sem a necessidade de abrir a cavidade abdominal, tendo recuperação mais rápida e menos traumática. Com o surgimento dessa nova técnica, surgiram também a necessidade e o desafio de treinar cirurgiões para adquirir as habilidades necessárias para sua prática, de forma eficiente e segura (SROKA et al, 2010)

Portanto, na cirurgia laparoscópica, a simulação adquiriu um papel fundamental no treinamento cirúrgico, tanto para treinar residentes quanto para cirurgiões que desejam treinar novas técnicas minimamente invasivas antes de sua implementação em pacientes, como a laparoscopia de porta única, cirurgia endoscópica transluminal de orifício natural (NOTES) ou incorporação de novas tecnologias e instrumentos laparoscópicos. A simulação laparoscópica pode até ser usada para treinamento básico durante uma carreira médica. A longa curva de aprendizado dessa técnica, juntamente com os altos riscos e custos de aprender técnicas complexas, como a sutura intracorpórea em pacientes, levaram ao desenvolvimento de centros de simulação especializados, permitindo que os cirurgiões aprendam com segurança e eficiência (VARAS et al, 2012; ORZECH,ET al, 2012)

Alguns estudos mostram que o treinamento de habilidades cirúrgicas antes da operação demonstrou um aprendizado mais eficiente na sala de operações, permitindo que o estagiário concentre a atenção nos detalhes técnicos do procedimento, sem a necessidade de aprender tudo isso pela primeira vez em um paciente (VAN SICKLE et al 2006; BRINDLEY et al, 2012) .

Com isso gerou a necessidade de saber quais são as melhores ou mais eficazes maneiras de aprender essas habilidades, valorizar avanços subsequentes na concepção do equipamento, e que representam efectivamente uma vantagem para levar a cabo técnicas que facilitam os procedimentos (ARKENBOUT et al, 2015; SAHU et al 2014).

Assim, criou-se o objetivo de estudo em avaliar o aprendizado de habilidades laparoscópicas básicas em estudantes de Medicina submetidos a treinamento em um simulador.

\section{MATERIAIS E MÉTODOS}

Trata-se de uma pesquisa de estudo Experimental - Prospectivo aberto e aplicado, como objetivo de avaliar a habilidade e destreza dos acadêmicos de medicina ao realizar vários exercícios no simulador de videolaparoscopia.

Os participantes foram 62 alunos que se dispuseram a participar, mas somente 30 
permaneceram no estudo completo, todos estes estudantes cursavam a partir do terceiro ao quinto ano do curso de Medicina na Universidade Central Del Paraguay, na cidade de Pedro Juan Caballero, departamento de Amambay.

A pesquisa foi realizada no período de março de 2019, no laboratório de habilidades morfofuncionais da Universidade Central Del Paraguay, no turno da tarde, com uma hora de duração para cada grupo, totalizando 6 horas de habilidades, em cada turno de treinamento, foram aceitos, no máximo 10 participantes, por termos somente 1 simulador.

Os alunos foram separados em grupos de treinamento, foram 3 grupos de 10 participantes em uma caixa simuladora por um período de 3 minutos por participantes no pré treinamento e 3 minutos nos pós treinamento com um total de 180 minutos em cada fase das habilidades.

O questionário coletava dados referentes a idade, sexo e qual a mão dominante e habilidade manual desenvolvida na prática.

A coleta de tempo e a pontuação anterior ao treinamento prático em simuladores dos alunos voluntários incluídos no estudo serviram como controle, no pré e pós habilidades para posterior resultados.

Os estudantes receberam orientações iniciais com noções sobre o manejo dos instrumentais e demonstração dos exercícios.
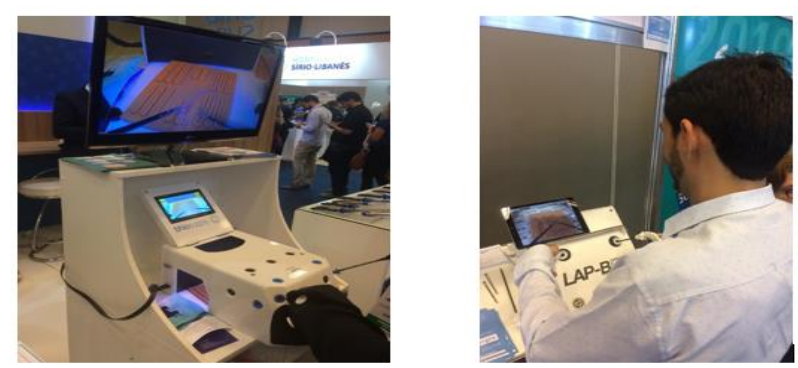

Figura 1.
O modelo experimental consistiu da utilização de 1 simuladores usado para treinamento em cirurgia laparoscópica (Figura 1). As habilidades avaliadas foram:

Pompons: Que consiste em introduzir uma serie de 30 pompons de pelúcia com um diâmetro de $1,5 \mathrm{~cm}$ com 3 cores diferentes em 3 respectivos recipientes, com diâmetro de $6 \mathrm{~cm}$ e uma altura de $3 \mathrm{~cm}$. (Fig 2).

Ligas: Colocado em uma plataforma de plástico quadrado $100 \mathrm{~cm}$ e 25 esporas emergem de sua superfície plástico com uma espessura de $3 \mathrm{~mm}$ e uma altura de $0,5 \mathrm{~cm}$ do mesmo material, o indivíduo foi instruído a acomodar 3 ligas elásticas com um comprimento de descanso de $3 \mathrm{~cm}$ acima destas esporas, formando figuras que um triangulo equilátero de $5 \mathrm{c}$ de lado, retângulo de $3,5 \times 7 \mathrm{~cm}$ uma linha reta de 9,5 cm de comprimento. (Fig 3).

Anéis: Esta atividade requer a colocação de 18 aneis de plástico em series de 3 cores, com $0,5 \mathrm{~cm}$ de espessura e Luz de $1 \mathrm{~cm}$ de diâmetro, em 3 colunas respectivas feito de madeira com ajustes exatos ao diâmetro dos anéis (Fig 4).

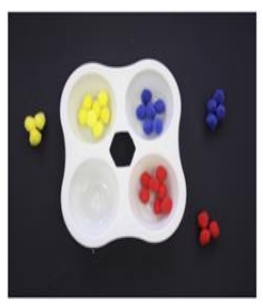

Figura 2.

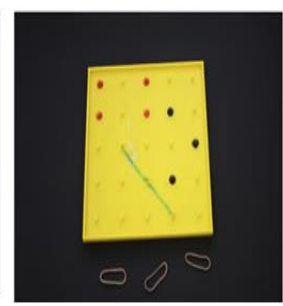

Figura 3.

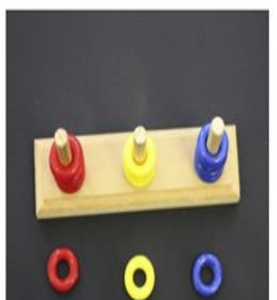

Figura 4.
Os critérios de inclusão foram somente alunos do terceiro ano ao quinto ano da Universidade Central Del Paraguai, todos de ambos os sexos, excluindo aqueles que tiveram um contato prévio com a cirurgia 
de vídeo-laparoscopia e tivesse alguma limitação morfofuncional para desempenho das atividades.

Os alunos voluntários preencheram um questionário demográfico e assinaram um Termo de Consentimento Livre e Esclarecido liberando suas informações para o estudo.

A coleta de dados foi realizada por meio de ficha experimental e suas habilidades realizadas. Os dados foram armazenados nos programas Microsoft Word e Microsoft Excel para análise. Os resultados foram apresentados em tabelas.

\section{Aspectos éticos}

Que conta com a aprovação do Comissão de Ética e Pesquisa baseado na Resolução no 3156/2006 e resolução 577/18 comprovado ao conselho nacional de educação superior no distrito do Paraguai e Departamento de Pedro Juan Caballero.

\section{RESULTADOS E DISCUSSÃO}

O estudo contou com a participação de 30 alunos com distribuição de sexo. Houve predominância de alunos do $3^{\circ}$ e $5^{\circ}$ ano da Universidade e com intenção futura de seguir carreira em cirurgia. As demais informações demográficas estão contidas na tabela 01 .

Tabela 01: Distribuição do perfil demográficos dos alunos submetidos as habilidades em simulação videolaparoscopico.

\begin{tabular}{lc}
\multicolumn{1}{c}{ Variáveis } & número $(\%)$ \\
\hline Idade, média & 21.6 \\
Sexo Masculino & $21(70 \%)$ \\
Sexo Feminino & $9(30 \%)$ \\
MÃO DOMINANTE &
\end{tabular}

$\begin{array}{cc}\text { Direita } & 27(90 \%) \\ \text { Esquerda } & 3(10 \%)\end{array}$

Fonte: Pesquisa Direta
Os tempos e pontuações obtidos foram analisados para verificar uma possível melhora póstreinamento. Na figura 5 , as pontuações de execução de cada exercício, medidos no pré e pós-treinamento, apresentaram evolução em todos os exercícios. O comparativo entre as duas etapas de treinamento obteve significância estatística $(\mathrm{p}<0,001)$, comparando os grupos, em fossemos colocar em ordem, o G2 foi vencedor, com pontuação de 90 pontos no final; seguido de $\mathrm{G} 2$ no pré treinamento teve 35 pontos, pós treinamento com 65 pontos e finaliza com grupo G1 com pré treino de 7 pontos, subindo para 60 pontos no pós treino.

Figura 5: Desempenho das habilidades laparoscópica em treinamento de transferências de pompons com estudantes de medicina do $3^{\circ}$ ao $5^{\circ}$ ano, com resultados de fases pré e pós treino, com tempo de 3 minutos em cada fase.

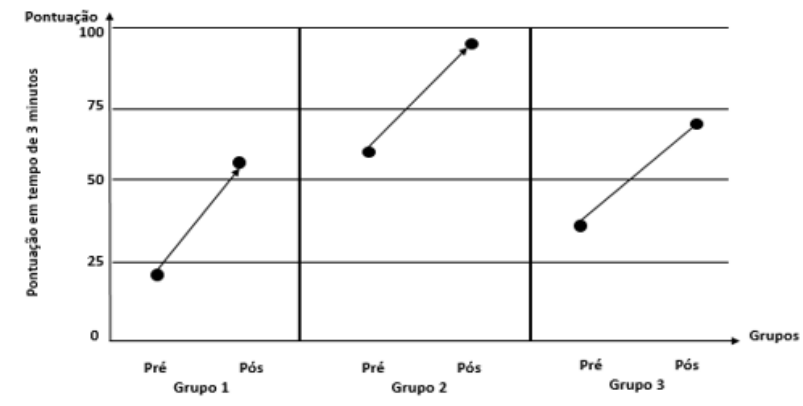

Já no exercício da liga em formar um triângulo em menor tempo está apresentada na figura 6. Houve uma variação de melhora de uma média geral dos três grupos no pré teve 159.33 segundos e pós treino uma média de 104.7 em segundo. Todas as pontuações apresentaram $\mathrm{p}<0,001$. Em individualidade, o G1 teve pré treino com tempo de 175 segundos e pós treinamento de 122 segundos; já o G2 com pre treino de 150 segundo e pós treinamento de 85 segundos, e finalizando o G3 teve pre treino de 153 segundo, nos pós treino um resultado de 107 segundo. A média geral saiu da soma dos índices dos grupos pré e pós treino. 
Figura 6: Desempenho das habilidades laparoscópicas na acomodação das ligas formando um triangulo, desenvolvidos por alunos do curso de medicina.

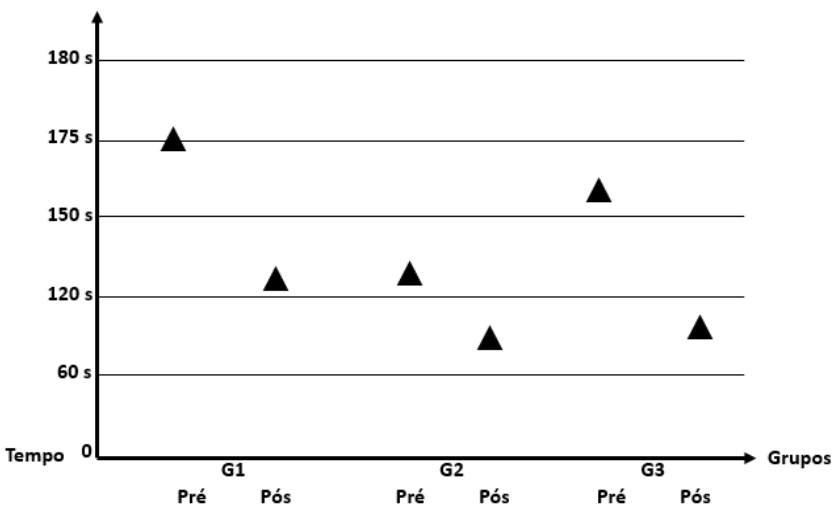

Na habilidade de desempenho no simulador laparoscópico para colocação de anéis no suporte, os seguintes resultados existiram, no G1 no pré treino foi alcançado 145 segundos e pós treino 90 segundos; já no G2 no pré treinamento foi alcançado 130 segundos e pós treino um valor de 68 segundos, no último grupo (G3) comparados aos outros teve tempo melhor tanto no pré e pós treino, com resultados significativos no préum tempo de 120 segundo e pós treino um tempo de 63 segundos sendo significativa para habilidades neste exercício, conforme figura 7 .

Figura 7: Tempo de execução de exercícios por estudantes de Medicina, no pré e pós-treinamento, em simulador cirúrgico laparoscópico na colocação de anéis no suporte.

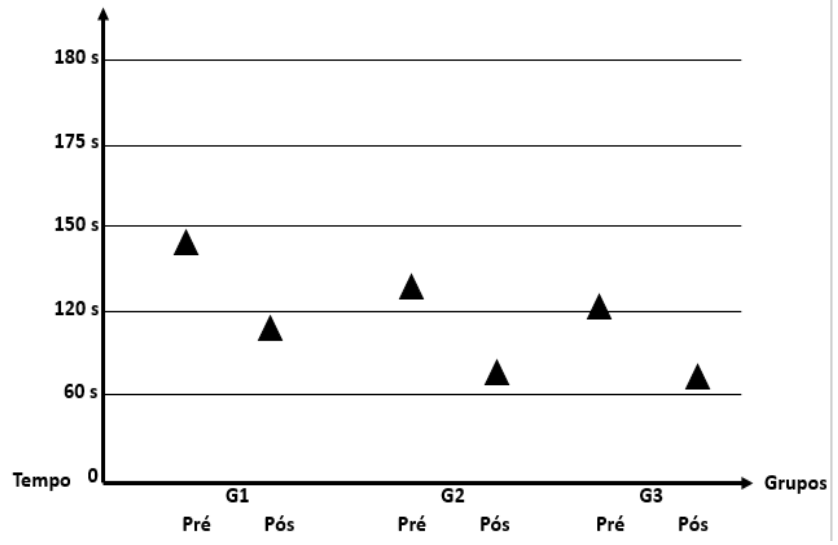

\section{DISCUSSÃO}

O achado importante deste estudo é a relação da habilidade de estudantes de Medicina que nunca foram expostos a prática de laparoscopia. Os alunos escolhidos foram aqueles que não tiveram a disciplina de técnica operatória podendo assim, oferecer dados verídicos sobre a habilidade. O desempenho dos alunos pré e pós-treino mostrou-se significativamente melhor para todos os exercícios. Com isso, mostra um contraposto à literatura, visto que a maioria dos estudos são realizados com médicos residentes.

Outro fator imprescindível é de descobrir os fatores que influenciem na aquisição de habilidade, qualificando, assim, o "melhor futuro cirurgião", seria de grande interesse para a educação cirúrgica, mas vale ressaltar que o desempenho do aluno no simulador de videolaparoscopia não significa necessariamente que este terá o mesmo desempenho de um cirurgião e não estará apto a realizar cirurgia.

Quanto a aprendizagem pelos estudantes participantes do programa de treinamento, a maioria considerou o treinamento satisfatório, o que mostra uma concordância com o desempenho observado.

Em proximidades ao estudo de Knorndorffer et al (2005) para o grupo de treinamento, o tempo médio para demonstrar a proficiência no simulador foi de 151 minutos (intervalo de 107 a 224 minutos) e o número médio de tentativas foi de 37 (intervalo de 24 a 51 tentativas). Tanto os grupos treinados quanto os de controle demonstraram melhora significativa na pontuação geral da linha de base. Mas o grupo treinado teve um desempenho significativamente melhor que o grupo controle no pós-teste (389 +/- 70 versus 217 +/$140, \mathrm{p}<0,001)$, confirmando a eficácia do treino, este estudo foi bem próximo do nosso, na figura 6 .

\section{CONCLUSÃO}

Acadêmicos de medicina que nunca foram expostos à laparoscopia apresentaram melhora do 
desempenho na realização de habilidades práticas em cirurgia laparoscópica mediante o treinamento em simuladores. Não foram identificados fatores e nem habilidades específicas dos estudantes que pudessem influenciar os resultados, independente do ano do curso, que não houve diferença estatísticas, todos obtiveram bom desempenho

\section{AGRADECIMENTO}

Agradecemos primeiramente a Deus por nos conceder forças para chegarmos até aqui, em seguida a Universidade Central Del Paraguay aos alunos pela oportunidade e disponibilidade em participar. Cada um serviu de base para tal realização deste estudo. E nosso orientador Dr. Marcelo Mendes Ribeiro pela contribuição e ensino. Não há conflito de interesse entre os autores.

Todos os autores declararam não haver qualquer potencial conflito de interesses referente a este artigo.

\section{REFERÊNCIAS}

AGGARWAL, Rajesh; DARZI, Ara. Simulação para aumentar a segurança do paciente: por que ainda não chegamos ?.Baú, v. 140, n. 4, pág. 854-858, 2011.

ANDERS ERICSSON, K. Prática deliberada e aquisição de atuação especializada: uma visão geral. Medicina de emergência acadêmica, v. 15, n. 11, pág. 988-994, 2008.
BRINDLEY, Peter G. et al. Simpósio anual da Associação Canadense de Cirurgiões Universitários. Simulação cirúrgica: a solução para um treinamento seguro ou uma promessa não cumprida ? : Victoria, BC, 10 de setembro de 2009. Canadian Journal of Surgery, v. 55, n. 4 Suplemento 2, pág. S200, 2012

CAVALINI, Worens Luiz Pereira et al. Desenvolvimento de habilidades laparoscópicas em estudantes de Medicina sem exposição prévia a treinamento cirúrgico. Einstein (São Paulo), v. 12, n. 4, p. 467-472, 2014.

ORZECH, Neil et al. Uma comparação de 2 currículos de treinamento ex vivo para habilidades laparoscópicas avançadas: um ensaio clínico randomizado. Anais de cirurgia, v. 255, n. 5, pág. 833-839, 2012.

REZNICK, Richard K .; MACRAE, Helen. Ensino de habilidades cirúrgicas - mudanças no vento. New England Journal of Medicine, v. 355, n. 25, pág. 2664-2669, 2006.

SROKA, Gideon et al. Os fundamentos do treinamento do simulador de cirurgia laparoscópica para a proficiência melhoram o desempenho laparoscópico na sala de cirurgia - um ensaio clínico randomizado. The American Journal of surgery, v. 199, n. 1, pág. 115120, 2010.

VARAS, Julián et al. Transferência significativa de habilidades cirúrgicas obtidas com um programa de treinamento laparoscópico avançado para uma jejunojejunostomia laparoscópica em um modelo suíno vivo: viabilidade de aprender laparoscopia avançada em uma residência em cirurgia geral. Endoscopia cirúrgica, v.26, n.12, pág.3486-3494,2012.

VAN SICKLE, Kent R .; RITTER, E. Matt; SMITH, C. Daniel. O novato pré-treinado: usando o treinamento baseado em simulação para melhorar o aprendizado na sala de cirurgia. Inovação cirúrgica , v. 13, n. 3, pág. 198-204, 2006. 